\title{
Pyoderma gangrenosum and suppurative hidradenitis association, overlap or spectrum of the same disease? Case report and discussion
}

\author{
GABRIELA TURCU ${ }^{1-3}$, ROXANA IOANA NEDELCU ${ }^{2,4}$, IULIA TEODORA NEDELCU ${ }^{3}$, \\ ANASTASIA HODOROGEA $^{3,4}$, MIHAELA BALABAN ${ }^{2,5}$, ALICE BRINZEA ${ }^{4,6}$, LUCIANA NICHITA ${ }^{7}$, \\ CRISTIANA GABRIELA POPP $^{7}$, SABINA ANDRADA ZURAC ${ }^{7}$ and RAZVAN THEODOR ANDREI ${ }^{7}$
}

\footnotetext{
${ }^{1}$ Department of Dermatology, Faculty of Medicine, Carol Davila University of Medicine and Pharmacy, 050474 Bucharest;

${ }^{2}$ Department of Dermatology, Derma $360^{\circ}$ Clinic, 011273 Bucharest; ${ }^{3}$ Department of Dermatology, Colentina Clinical Hospital, 020125 Bucharest; ${ }^{4}$ Department of Pathophysiology, Faculty of Medicine,

Carol Davila University of Medicine and Pharmacy, 020105 Bucharest; ${ }^{5}$ Department of Biochemistry, Faculty of Medicine, Carol Davila University of Medicine and Pharmacy, 050474 Bucharest; ${ }^{6}$ Department of Ambulatory, National Institute for Infectious Diseases Prof. Dr. Matei Balș, 020105 Bucharest;

${ }^{7}$ Department of Pathology, Colentina Clinical Hospital, 020125 Bucharest, Romania
}

Received September 5, 2019; Accepted October 7, 2019

DOI: $10.3892 /$ etm.2019.8150

\begin{abstract}
Suppurativehidradenitis and pyodermagangrenosum are rare disorders that can be seen isolated or even more rare, as part of different autoinflammatory syndromes: Pyoderma gangrenosum, acne, and hidradenitis suppurativa (PASH), pyoderma gangrenosum, acne, pyogenic arthritis, and hidradenitis suppurativa (PAPASH) or psoriatic arthritis, pyoderma gangrenosum, acne, and hidradenitis suppurativa (PsAPASH). Although they have different clinical features, suppurative hidradenitis and pyoderma gangrenosum seem to share similar pathogenic pathways involving a dysregulated innate immune system, with neutrophilic inflammation, mediated by IL- $1 \beta$, controlled by NALP 3 inflammasome pathway. We report a case of a 53-year-old male patient previously diagnosed with HS in inguinal-scrotal area that developed rapidly after a traumatic injury on his left anterior calf, a painful inflammatory plaque with pustules on the surface that rapidly progressed (24-48 h) to form ulcers. The lesions ended up healing with a large scarring plaque with cribriform openings, multiple fibrous bridges, open comedones, and double-ended pseudo-comedones. Although the clinical aspect at presentation together with the aspect on the first biopsy were suggestive for pyoderma
\end{abstract}

Correspondence to: Dr Roxana Ioana Nedelcu, Department of Pathophysiology, Faculty of Medicine, Carol Davila University of Medicine and Pharmacy, 1 Calistrat Grozovici Street, 020105 Bucharest, Romania

E-mail: roxanaioana.nedelcu@yahoo.com

Key words: pyoderma gangrenosum, hidradenitis suppurativa gangrenosum, the healing aspect is more commonly seen in suppurative hidradenitis. Commonly seen in acne, in the healing phase of suppurative hidradenitis but more rarely in pyoderma gangrenosum, the formation of comedones seem to be a complex process and raise the question if these entities represent in our patient an association, an overlap or the spectrum of the same disease.

\section{Introduction}

Suppurative hidradenitis ( $\mathrm{SH})$ is a chronic, relapsing, inflammatory condition, usually with insidious onset, affecting typically flexural areas such as the axillary, inguinal and anogenital region, with a variable clinical presentation, inflamed nodules, suppurating sinus tracts, scarring and secondary contracture $(1,2)$.

Genetic, immunological and environmental factors (infectious agents, smoking, adipose tissue, friction) favor hair follicle occlusion, accumulation of cellular debris and cyst formation, followed by follicular rupture, inflammation and chronic suppuration (3). When the deep part of the hair follicle is damaged, it will result in open comedones, frequently grouped and interconnecting $(4,5)$. Reepithelization of the follicles will form sinus tracts and areas of confluent nodular lesions will heal with inter-communicating sinuses and fistulas, punctate, acneiform, atrophic or hypertrophic scars and fibrotic bridges $(2,4)$.

Pyoderma gangrenosum (PG) is a neutrophilic dermatosis, an inflammatory and ulcerative disorder of the skin with rapid and painful onset of inflammatory papules, pustules, vesicles or nodules that form erosions or ulcers that heal with atrophic, cribriform scars, commonly localized on lower extremities and trunk (6). Genetic and immunological factors (dysregulation of the innate immune system, neutrophil dysfunction) 


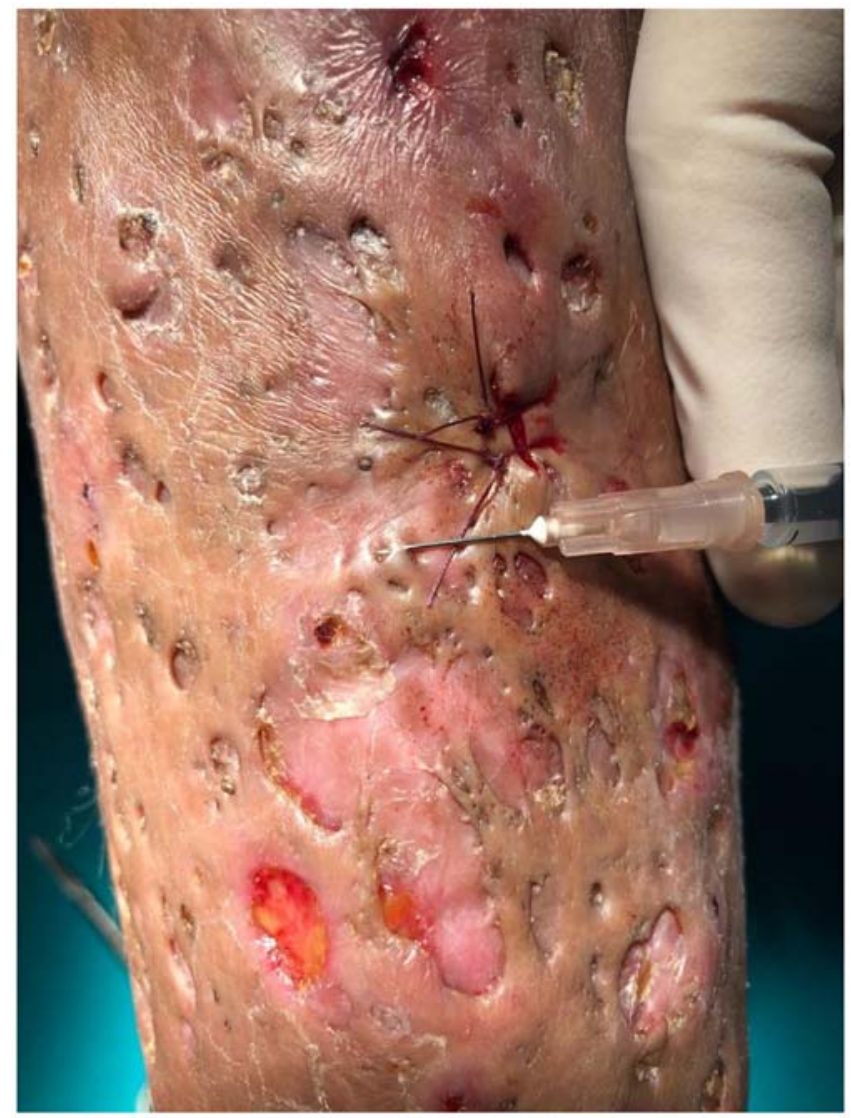

Figure 1. Left anterior calf - multiple fibrous bridges, open comedones, double-ended pseudo-comedones on the surface and several painful ulcers.

induce perifollicular inflammation and intradermal abscess and later epidermal and superficial dermal necrosis with an underlying mixed inflammatory cell infiltrate, with or without vasculitis (7).

Despite clinical differences, PG and SH seem to share similar pathogenic pathway (8). Understanding the inflammatory process plays an essential role in cutaneous pathology, in both inflammatory diseases and tumorigenesis $(9,10)$. HS, PG and cystic acne can be present in the same patient as part of pyoderma gangrenosum, acne and suppurative hydradenitis (PASH) an autoinflammatory syndrome characterized by neutrophilic inflammation, mediated by IL-1 $\beta$, controlled by NALP3 inflammasome pathway (8).

\section{Case report}

We report a case of a 53-year-old male patient, normal weight, 16.5-pack-year smoker, referred to the Dermatology Department for a large scarring plaque of $\sim 20 \times 8 \mathrm{~cm}$ on his left anterior calf, with cribriform openings, multiple fibrous bridges, open comedones, double-ended pseudo-comedones on the surface and several painful ulcers of $0.5-2 \mathrm{~cm}$ in diameter, with undefined, violaceous borders and seropurulent, sanguinous discharge (Fig. 1). Consent for publication was obtained from the patient.

The lesion first appeared 2 months prior to consultation, after a traumatic injury, with a very painful onset of an inflammatory plaque with pustules on the surface that rapidly

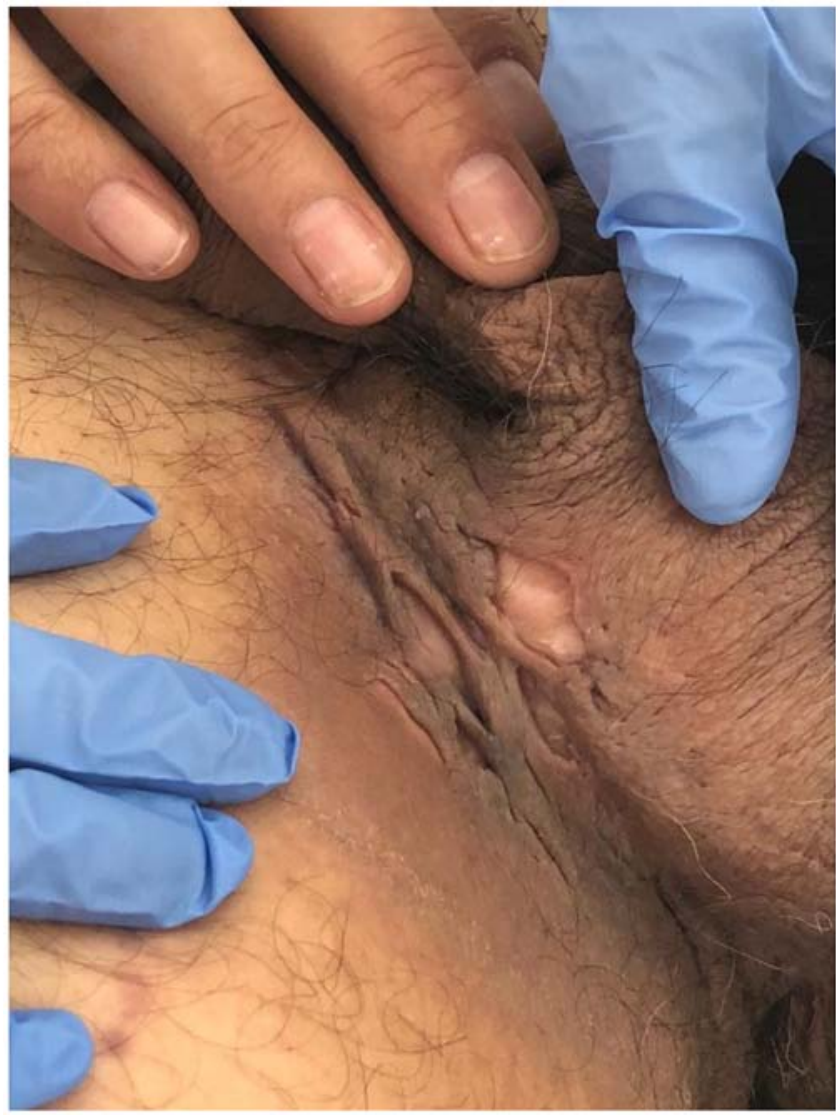

Figure 2. Inguinal-scrotal area - retractile, fibrotic scars, depressed scars, hyperpigmentation and open comedones

progressed (24-48 h) to form ulcers. The patient self treated with 2 courses of a 10-day oral ampicillin with no signs of improvement. Therefore he had been hospitalized in the Infectious Diseases Department. Laboratory workup revealed increase of inflammatory markers and serology for hepatitis infections, HIV and syphilis, fungal culture and PCR for BK were all negative. Bacterial cultures from multiple sites showed growth of Escherichia coli, Enteroccocus faecalis and Peptophilus spp. Lower limb X-ray showed heterogeneous soft tissue swelling. Treatment was undertaken with ceftriaxone and vancomycin and switched to amoxicillin/clavulanic acid and vancomycin, as the antimicrobial susceptibility tests showed resistance to ceftriaxone, for 20 days, with clinical improvement. Biopsy specimen from the border of the leg ulcer revealed neutrophilic inflammation and areas of necrotic and fibrotic connective tissue consistent with ulcerative PG and the patient was referred to the dermatology clinic.

Physical examination also revealed a bilateral, inguinal-scrotal area, fibrotic scar tissue, depressed scars, hyperpigmentation and open comedones, compatible with the diagnosis of HS (Fig. 2). The patient underwent surgery for relapsing left and right inguinal abscess in 2014 and 2016. No acne scars were identified on the face. Laboratory workup revealed decrease of inflammatory markers and increased liver enzymes, that normalized rapidly in the following days (probably drug induced). No pathergy sign. Medical investigations did not reveal signs of inflammatory bowel disease, hematologic malignancy or arthritis. 


\section{Results}

Due to the atypical healing pattern, with the predominance of comedo-like openings, double-ended pseudocomedones and bridging fibrosis, a new biopsy was taken which showed fistulous cystic tract associated with fibrotic tissue. These aspects are most commonly met in HS, but also in primary infection, ruptured epidermal cyst, pilonidal sinus, acne vulgaris, Favre-Racouchot disease, syringoma, tricoepithelioma, nevus comedonicus or vegetative PG $(6,11)$. Multiplicity, no evidence of microorganisms on special stains and clinical correlation were suggestive for the diagnosis of late stage of suppurative hidradenitis.

Systemic treatment with Sulfasalazine $500 \mathrm{mg}$ once daily for one week, then increasing the dose weekly, until $2 \mathrm{~g} /$ day and topical potent steroids controlled the inflammation satisfactorily, few small ulcers still continuing to appear occasionally, but without accompanying pain.

\section{Discussion}

We had 3 questions regarding the lesion from the leg: Is this a typical PG but with atypical healing pattern? Is this an ectopic HS in a male patient with typical HS lesion in inguinal area? Is this a lesion with elements of both PG and HS?

The abrupt onset of the painful lesion, after local trauma, with pustule that rapidly form ulceration, with violaceous border, together with the pathological aspect and the lack of criteria for other causes of ulceration are all suggestive for PG. But regarding the presence of double-ended pseudo-comedones, Revuz and Jemec (12), considered them a typical sign of HS, formed after reepithelisation of 2 adjacent destroyed follicles (13). Boer and Jemec (14) studied pseudo-comedo formation in HS and showed that they appear secondary to hair follicle damage. Although inflammation in PG starts perifollicularly, healing with pseudo-comedones is not a feature described in PG. Sinus tract formation can be consistent with PG, but the vegetative subtype and this was not the case (6). Although not mentioned in the text, in one of the four cases of PASH presented by Join-Lambert et al (15), as seen in the pictures, the lesions of ulcerative PG healed with open comedones.

There are few studies of cases of chronic cutaneous lupus erythematosus healing with pseudo-comedones and we can hypothesize that foliculocentric inflammation is responsible also in these situations for the pseudo-comedones formation, but still, it rarely happens (16).

Regarding the second question, the scarring aspect of the plaque on the leg, in a patient known with HS in inghinoscrotal area, was suggestive for ectopic HS, as well as the aspect of the second biopsy, as in the case described by Rondags et al (17), but the rapid onset of the lesions is not compatible with the diagnosis of HS.

So we appreciate that the lesion on the leg had features of both PG and HS, proving the link between the two diseases, probably through mechanisms not yet elucidated.

The differential diagnosis issues we highlighted in the presented case also permitted us to speculate that the association between HS, PG and acne in syndromes such as PASH may have a deeper understanding. Does the association of the three diseases constitute a syndrome or is there a single disease in which one, two or all three manifestations may be observed in the same patient?

We consider that the study of comedogenesis remains one of the essential objectives for elucidating the mechanisms involved in diseases such as pyoderma gangrenosum, supurative hydradenitis and acne. Interestingly, as an easily clinically recognizable mark by adolescents and cosmetics experts, for dermatologists and researchers, the comedo is still an incomplete elucidated mystery regarding the biological events involved in its formation. The complexity of the comedo's significance also arises from the different roles that it seems to play in various diseases: in acne, microcomedo formation is the essential element for the development of acne lesions; in HS the comedo is formed in the active phases of the disease when the deep part of the follicle is affected; in diseases such as PG, comedo may be an aspect of healing phases, through poorly understood mechanisms, probably also as a consequence of follicular destruction.

\section{Acknowledgements}

Not applicable.

\section{Funding}

This study is supported by grants of Ministery of Research and Innovation, CNCS-UEFISCDI, project number PN-III-P4-ID-PCE-2016-0641, within PNCDI III and CCCDI-UEFISCDI, project number 61PCCDI/2018 PN-III-P 1-1.2-PCCDI-2017-0341, within PNCDI-III.

\section{Availability of data and materials}

All patient data are mentioned in the article and are available from the corresponding author on resonable request.

\section{Authors' contributions}

GT, RIN, ITN, AH, MB and AB made substantial contributions to the conception and the acquisition of data for the study. LN, CGP, SAZ and RTA made substantial contributions to the analysis and interpretation of data for the work. All the authors revised critically for important intellectual content, approved the final version to be published and agreed to be accountable for all aspects of the study in ensuring that questions related to the accuracy or integrity of any part of the work are appropriately investigated and resolved.

\section{Ethics approval and consent to participate}

Patient consent to participate was obtained.

\section{Patient consent for publication}

Patient consent for the publication of the images was obtained before publication.

\section{Competing interests}

The authors declare that they have no competing interests. 


\section{References}

1. Kurzen H, Kurokawa I, Jemec GBE, Emtestam L, Sellheyer K, Giamarellos-Bourboulis EJ, Nagy I, Bechara FG, Sartorius K, Lapins J, et al: What causes hidradenitis suppurativa? Exp Dermatol 17: 455-472, 2008.

2. Ingram JR: Hidradenitis suppurativa: Pathogenesis, clinical features, and diagnosis. UpToDate 2018 (Jan 1, 2018). Available from: https://www.uptodate.com/contents/hidradenitis-suppurativa-pathogenesis-clinical-features-and-diagnosis

3. von Laffert M, Stadie V, Wohlrab J and Marsch WC: Hidradenitis suppurativa/acne inversa: Bilocated epithelial hyperplasia with very different sequelae. Br J Dermatol 164: 367-371, 2011.

4. Habif T: Clinical Dermatology: A Color Guide to Diagnosis and Therapy. Mosby, New York, NY, 2004.

5. Desai N, van der Zee HH and Jemec GB: Hidradenitis Suppurativa. In: Rook's Textbook of Dermatology. Griffiths CE, Barker J, Bleiker T, Chalmers R, Creamer D (eds). 9th edition. Wiley Blackwell, pp90.1-90.66, 2016.

6. Schadt C: Pyoderma Gangrenosum: Pathogenesis, Clinical Features, and Diagnosis. 2018. Available from: https://www. uptodate.com/contents/pyoderma-gangrenosum-pathogenesisclinical-features-and-diagnosis\#H603259

7. Ahronowitz I, Harp J and Shinkai K: Etiology and management of pyoderma gangrenosum: A comprehensive review. Am J Clin Dermatol 13: 191-211, 2012.

8. Braun-Falco M, Kovnerystyy O, Lohse $\mathrm{P}$ and Ruzicka T: Pyoderma gangrenosum, acne, and suppurative hidradenitis (PASH) - a new autoinflammatory syndrome distinct from PAPA syndrome. J Am Acad Dermatol 66: 409-415, 2012.
9. Antohe M, Nedelcu R, Nichita L, Popp C, Cioplea M, Brinzea A, Hodorogea A, Calinescu A, Balaban M, Ion DA, et al: Tumor infiltrating lymphocytes: The regulator of melanoma evolution (Review). Oncol Lett 17: 4155-4161, 2019.

10. Cioplea M, Caruntu C,Zurac S, Bastian A, Sticlaru L, Cioroianu A, Boda D, Jugulete G, Nichita L and Popp C: Dendritic cell distribution in mycosis fungoides vs. inflammatory dermatosis and other T-cell skin lymphoma. Oncol Lett 17: 4055-4059, 2019.

11. Calonje E, Brenn T and Lazar A: Neutrophilic and eosinophilic dermatoses. In: McKee's Pathology of the Skin. 4th edition. McKee PH (ed). Elsevier Limited, p657, 2012.

12. Revuz JE and Jemec GB: Diagnosing hidradenitis suppurativa. Dermatol Clin 34: 1-5, 2016.

13. Margesson LJ and Danby FW: Hidradenitis suppurativa. Best Pract Res Clin Obstet Gynaecol 28: 1013-1027, 2014.

14. Boer J and Jemec GB: Mechanical stress and the development of pseudo-comedones and tunnels in Hidradenitis suppurativa/Acne inversa. Exp Dermatol 25: 396-397, 2016.

15. Join-Lambert O, Duchatelet S, Delage M, Miskinyte S, Coignard H, Lemarchand N, Alemy-Carreau M, Lortholary O, Nassif X, Hovnanian A, et al: Remission of refractory pyoderma gangrenosum, severe acne, and hidradenitis suppurativa (PASH) syndrome using targeted antibiotic therapy in 4 patients. $\mathrm{J}$ Am Acad Dermatol 73 (Suppl 1): S66-S69, 2015.

16. Farias DF, Gondim RM, Redighieri IP, Muller H and Petri V: Comedonic lupus: a rare presentation of discoid lupus erythematosus. An Bras Dermatol 86 (Suppl 4): S89-S91, 2011.

17. Rondags A, Diercks GF, Werker PMN, Jonkman MF and Horváth B: Ectopic hidradenitis suppurativa on the dorsal foot of a road maker. JAAD Case Rep 3: 429-431, 2017. 